\title{
Relative usefulness of electron microscopy and immunocytochemistry in tumour diagnosis: 10 years of retrospective analysis
}

\author{
A U H Dar, P M Hird, B E Wagner, J C E Underwood
}

\begin{abstract}
Aims: To determine retrospectively the relative usefulness of electron microscopy and immunocytochemistry for tumour diagnosis; to monitor the influence of new antibodies and antisera on the use of these techniques in one laboratory.

Methods: During 1980 to 1989 inclusive, 726 tumours were examined by electron microscopy, 862 by immunocytochemistry, and 286 by both techniques. The choice of techniques and, for immunocytochemistry, the range of antibodies used were compared between each category of final diagnosis.

Results: During the study period there was a sharp fall in the use of electron microscopy and a corresponding rise in immunocytochemistry. These trends applied to all categories of final tumour diagnosis, but the use of electron microscopy was sustained longer for lesions suspected or eventually confirmed to be melanomas or amine precursor uptake decarboxylation cell carcinoma (APUDomas)-for example, carcinoid tumours. The immunocytochemistry:electron microscopy use ratios ranged from $2 \cdot 07: 1$ to $0 \cdot 44: 1$ for the categories in which lymphoma and APUDoma, respectively, were the final diagnoses. The abandonment of electron microscopy for suspected or confirmed lymphomas and carcinomas corresponded to the increasing availability of relevant antisera and antibodies.

Conclusions: The wider application of immunocytochemistry for tumour diagnosis is endorsed, but electron microscopy should be retained for selected cases in which the results of immunocytochemistry might be predictably ambiguous or otherwise unhelpful.
\end{abstract}

Most tumours can be identified histologically by routine light microscopy using a limited repertoire of special stains. However, since the 1960s, electron microscopy has been used in many centres ${ }^{1-3}$ and, since the 1970 s, immunocytochemistry has become increasingly popular. ${ }^{4-6}$ Although these techniques allow tumours to be typed accurately from their ultrastructural features, or from their immunocytochemical profile, there have been very few systematic studies comparing the relative diagnostic usefulness of these methods that would provide an informed basis for their more selective future use.
The aims of this study were to analyse retrospectively how electron microscopy and immunocytochemistry had been used for tumour diagnosis in one histopathology department over a 10 year period. During the period 1980-89 inclusive, many new antibodies and antisera became available and the influence of these on electron microscopy use was determined. Two years have elapsed since the end of the study period, during which the clinical behaviour of the tumours has not caused the final diagnoses to be reviewed.

This type of study can only be performed retrospectively, otherwise the histopathologists' use of these techniques during the study period might have been influenced by the knowledge that their behaviour would be analysed.

\section{Methods}

The files of the Department of Histopathology, Royal Hallamshire Hospital, Sheffield, were searched for the accession period 1 January 1980 to 31 December 1989 to recover details of all tumour cases on which either electron microscopy or immunocytochemistry had been performed for diagnostic purposes. The case material was derived from all body systems apart from the female genital tract and the central nervous system; paediatric lesions were also excluded.

Most samples for electron microscopy were taken, subsequent to light microscopy, from tissue that had been fixed routinely in $10 \%$ formalin. A few samples from formalin fixed, small biopsy specimens were taken from paraffin wax blocks. The samples were post-fixed in osmium tetroxide, dehydrated, embedded in resin, and thick sections were stained with toluidine blue to facilitate selection of blocks and areas within them for electron microscopy. Thin sections were stained with uranyl acetate and lead citrate and examined in an AEI Corinth transmission electron microscope.

With the exception of suspected lymphomas, where frozen $\left(-70^{\circ} \mathrm{C}\right)$ unfixed tissue was usually available, immunocytochemistry was performed on paraffin wax sections of formalin fixed tissue. Optional proteolysis and blockage of endogenous peroxidase were performed when appropriate. Various immunoperoxidase methods were used with diaminobenzidine as the chromogen: an indirect method was used until 1986 when the peroxidase-antiperoxidase (PAP; mouse) method was instituted; an avidin-biotin complex (ABC) method was
J C E Underwood

Correspondence to: Professor J C E Underwood Accepted for publication 5 February 1992 
Table 1 Comparison of usage of electron microscopy or immunocytochemistry in each category of final tumour diagnosis (1980-1989 inclusive)

\begin{tabular}{lllll}
\hline & \multicolumn{2}{l}{ Number of tumours } & & \\
\cline { 2 - 5 } $\begin{array}{l}\text { Tumour } \\
\text { category }\end{array}$ & Immunocytochemistry & Electron microscopy & $\begin{array}{l}\text { Immunocytochemistry } \\
\text { and electron microscopy }\end{array}$ & $\begin{array}{l}\text { Immunocytochemistry: } \\
\text { electron microscopy ratio }\end{array}$ \\
\hline Lymphoma & 346 & 167 & 105 & $2 \cdot 07$ \\
Carcinoma & 264 & 217 & 72 & $1 \cdot 2$ \\
Sarcoma & 144 & 178 & 69 & $0 \cdot 81$ \\
Melanoma & 18 & 27 & 13 & $0 \cdot 66$ \\
APUDoma & 23 & 52 & 20 & $0 \cdot 44$ \\
\hline
\end{tabular}

used from 1987. In every case an antibody to factor VIII related antigen (expressed by vascular endothelium) was used as a positive control and to determine optimum proteolysis.

In each case the final diagnosis was recorded together with a summary of the techniques and immunological reagents used in each case. In a smaller sample of 60 cases, representative of each of the major categories of tumour diagnosis (carcinoma, lymphoma, sarcoma, melanoma, and APUDoma, including carcinoids), the original ultrastructural and immunocytochemical material was reviewed in detail to judge which of the techniques made the greater contribution to the final diagnosis.

\section{Results}

ELECTRON MICROSCOPY AND

IMMUNOCYTOCHEMISTRY USE RATES (1980-89)

During $1980-89, \ldots 1302$ tumours were subjected to diagnostic electron microscopy or immunocytochemistry; 726 were examined by electron microscopy, 862 by immunocytochemistry; and 286 by both techniques.

During this period there was a sharp fall in the number of cases examined by electron microscopy and a commensurate rise in immunocytochemistry use (fig 1). These trends applied to all categories of final tumour diagnosis, although the conversion from electron microscopy to immunocytochemistry as the preferred technique occurred slightly ear-

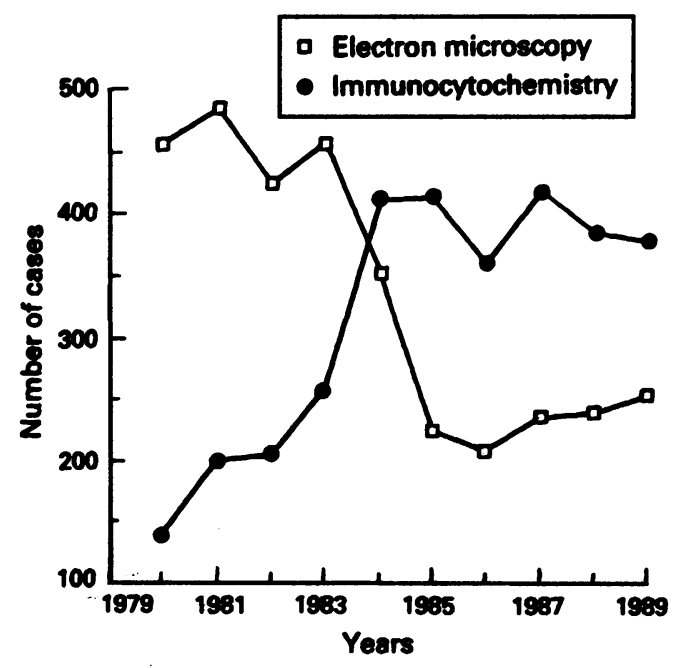

Figure 1 Comparison of usage of electron microscopy and immunocytochemistry for tumour diagnosis (1980-1989 inclusive). lier for lymphomas (1983) than for carcinomas and sarcomas (1984). For APUDomas (including carcinoids) and melanomas, the rates of use of the two techniques merged in about 1986 and have continued approximately in parallel thereafter.

\section{TUMOUR CATEGORIES}

Data for the entire study period were aggregated to reveal the preferred technique for the total cases in each tumour category (table 1). For lymphomas the immunocytochemistry: electron microscopy use ratio was $>2: 1$. Conversely, for APUDomas (including carcinoids), the ratio was reversed at $<0 \cdot 5: 1$. For carcinomas and sarcomas, the ratios were $1 \cdot 2: 1$ and $0 \cdot 8: 1$, respectively.

ANTIBODY USE

For each case in which immunocytochemistry had been performed, the antibodies and antisera used were recorded. The results reflect both their availability and perceived diagnostic utility reinforced by experience. Two patterns of usage emerged.

Some antisera, exemplified by those to $a$-1-antitryspin and lysozyme (fig 2), were much used initially during the study period as markers of histiocytic lineage. From the mid-

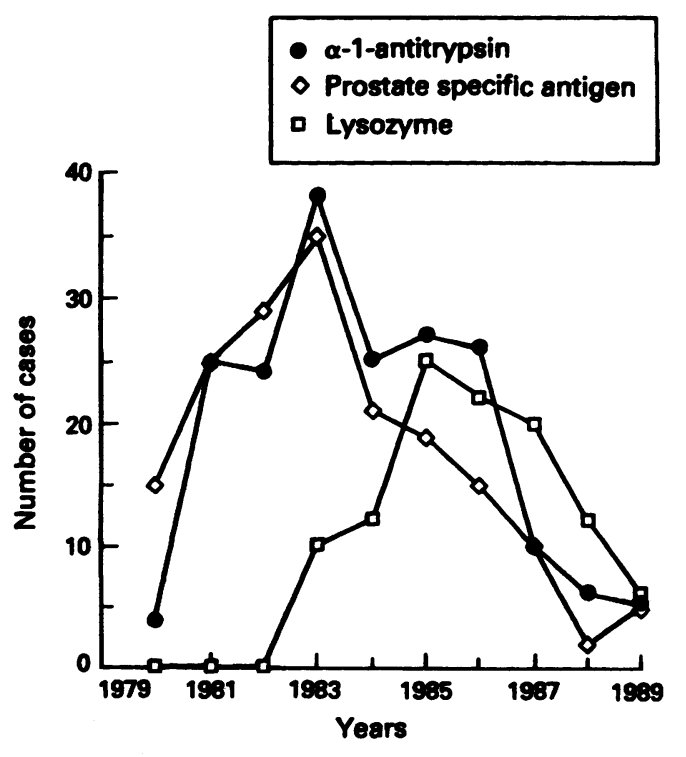

Figure 2 Antibodies/antisera exemplifying declining use for tumour diagnosis. The reduction in use corresponds to the availability of more reliable markers; for example, a-1-antitrypsin and lysozyme as macrophage markers have been superceded by a-1-antichymotrypsin, M387 and CD68 antibodies. 
Table 2 Influence of electron microscopy and immunocytochemistry on the final diagnosis in 60 selected tumours, representing major categories, that had been examined by both techniques

\begin{tabular}{llll}
\hline Tumour category & Number of tumours & $\begin{array}{l}\text { Positive } \\
\text { immunocytochemistry * (\%) }\end{array}$ & $\begin{array}{l}\text { Negative immunocytochemistry but } \\
\text { diagnostic ultrastructure (\%) }\end{array}$ \\
\hline Lymphoma & 19 & $14(74 \%)$ & $4(21 \%)$ \\
Carcinoma & 17 & $14(82 \%)$ & $3(18 \%)$ \\
Sarcoma & 11 & $4(36 \%)$ & $3(27 \%)$ \\
Melanoma & 4 & $3(75 \%)$ & $1(25 \%)$ \\
APUDoma & 9 & $7(78 \%)$ & $2(22 \%)$
\end{tabular}

¿Using one or more relevant antisera/antibodies

1980s their use declined so that by 1989 they were each used in only five cases. Other reagents showing a similar but less sharp decrease in use were those for carcinoembryonic antigen, $a$-fetoprotein, prostate specific antigen and, latterly, desmin and vimentin.

The other pattern of use, exemplified by antibodies to epithelial cytokeratins, S100, leucocyte common antigen (CD45), and lymphocyte markers such as UCHL1 (CD45RO), showed a rapid rise following their addition to the repertoire during the $1980 \mathrm{~s}$, reaching a sustained plateau within a few years (fig 3 ).

\section{ANALYSIS OF SAMPLE CASES}

Sixty cases, on which both electron microscopy and immunocytochemistry had been performed, and which were representative of each tumour category, were chosen for detailed appraisal (table 2).

Of the 17 carcinomas, 14 showed positive immunostaining for epithelial markers, but all 17 had diagnostic or confirmatory features (such as desmosomes) on electron microscopy. Similarly, all 13 cases of melanoma and APUDoma had diagnostic features on electron microscopy, but five of them were negative for S100 on immunocytochemistry. Eighty percent of lymphomas were positive for leucocyte common antigen, with other markers frequently present according to the type of lesion; electron microscopy was of value only in excluding the presence of features, such as desmosomes, melanosomes, that might favour an alternative diagnosis.

\section{RELATIVE COSTS}

The relative technical costs of using electron microscopy and immunocytochemistry for tumour diagnosis during 1980-89 was estimated from Welcan unit data. For each case, on average, it was estimated that electron microscopy amounted to 449 Welcan units and immunocytochemistry to 340 Welcan units. However, these are mean values and, in the latter instance, will vary per case according to the range of antibodies used. The actual costs of the two techniques could not be determined from the Welcan unit values due to the complexities of inflation, a series of budgetary adjustments during the study period, the effect of capital costs, and pathologists' unaccounted time.

\section{Discussion}

Electron microscopy and immunocytochemistry have undoubtedly contributed to the improved precision of tumour diagnosis. The number of cases classified as "anaplastic tumour" or "undifferentiated neoplasm" diminished substantially because, using these techniques, subtle or otherwise invisible markers denoting a specific histogenesis can be shown.

Our results from this retrospective analysis commend the use of both techniques in selected cases. Electron microscopy is the most useful in those cases in which the histological pattern favours a diagnosis of APUDoma or melanoma; immunocytochemistry is often helpful, but the ultrastructural evidence of neurosecretory type granules or melanosomes is very compelling and they are robust enough to withstand suboptimal processing. This contrasts with suspected lymphomas in which immunocytochemistry distinguishes these lesions from other categories of neoplasm - for example, usually by virtue of leucocyte common antigen (CD45) staining - but it also has the potential to classify further the disease by using a panel of antibodies recognising $B$ cell and $T$ cell markers and cells of histiocytic lineage.

For sarcomas and spindle-cell neoplasms of uncertain type, electron microscopy is a valuable technique. ${ }^{7-9}$ Epithelial cytokeratin immunostaining is frequently misleadingly positive in tumours finally classified as smooth muscle or endothelial neoplasms ${ }^{10-12}$; electron

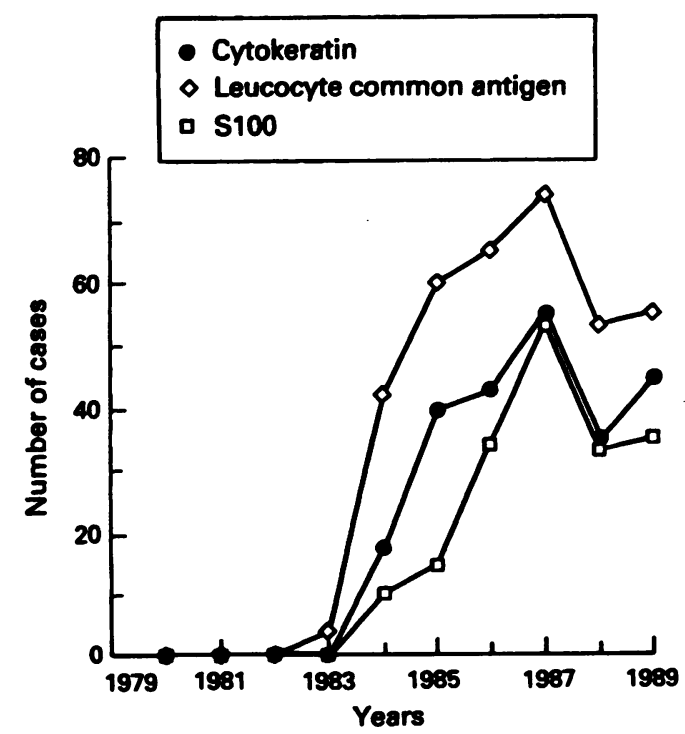

Figure 3 Antibodies/antisera exemplifying increasing usage for tumour diagnosis. The data for cytokeratin antibody usage were aggregated for all specificities-for example, PKK1, CAM 5.2. 
microscopy may well be diagnostic by demonstrating specific features such as myofilaments with characteristic dense bodies in smooth muscle tumours, but the use of an antibody panel can avoid the risk of misinterpreting the clinical relevance of cytokeratin immunostaining.

Since the end of the study period new reagents have further enhanced the diagnostic utility of immunocytochemistry, such as $Q$ BEND-10 and CD31 antibodies for endothelial differentiation, NKB45 and NK1C3 for melanocytic differentation, and AUA1 and BerEP4 for differentiating adenocarcinoma from mesothelioma. Chromogranin antibodies almost certainly increased the utility of immunocytochemistry for diagnosing APUDomas towards the end of the review period. Immunocytochemistry continues to be invaluable for classifying precisely the malignant lymphomas for which electron microscopy has very limited capacity.

Comparison of electron microscopy and immunocytochemistry on the basis of Welcan units is of limited value because this method of workload measurement does not cater for the pathologists' time or cognitive input, nor does it allow for the greater capital and recurrent costs of an electron microscope (at least under the budgetary arrangements in place during the study period). The pathologists' input may be greater for electron microscopy than for immunocytochemistry, because the former requires careful and time consuming selection of tissue areas and image fields for interpretation. Whereas many immunocytochemical preparations can be deemed positive or negative in the relevant cells after a relatively brief inspection, the interpretation of electron micrographs often requires more judgment.

Another difference between the two techniques affecting their usefulness is that with immunocytochemistry a limited number of antibodies and antisera has to be selected specially for each case and each reagent has relatively narrow diagnostic potential; the choice should be made rationally. ${ }^{13}$ This contrasts with electron microscopy where no specific question has to be framed other than the need to ensure that the small sample that is processed is representative; the range of ultrastructural features that may be disclosed by this technique covers a very wide range of diagnostic possibilities. Sampling is less of a problem with immunocytochemistry; indeed, distinct advantages of this technique include its ability to detect small numbers of cells either confined to one area of the tissue, such as micrometastasis, or camouflaged by an inflammatory infiltrate.

We conclude that in cases in which light microscopy favours melanoma or APUDoma electron microscopy is more likely to yield unequivocal diagnostic information. If the histological pattern favours either lymphoma or carcinoma immunocytochemistry using appropriate primary reagents is the method of first choice. For spindle-cell lesions and other histological patterns favouring a diagnosis of sarcoma, both immunocytochemistry and electron microscopy offer complementary data.

We are grateful to $\mathrm{Mr}$ G Anderson for his help with workload calculations and to numerous colleagues who made use of electron microscopy and immunocytochemistry for tumour diagnosis during the study period.

1 Mackay B, Silvia EG. Diagnostic electron microscopy in oncology. Pathol Annu 1980;15:241-69.

2 William MJ, Uzman BG. Uses and contributions of diagnostic electron microscopy in surgical pathology. Hum Pathol 1984;15:738-45.

3 Fisher C, Ramsay AD, Griffiths $M$, McDougall J. An assessment of the value of electron microscopy in tumour diagnosis. $f$ Clin Pathol 1985;38:403-8.

4 Gatter KC, Alcock C, Heryet A, Mason DY. Clinical importance of analysing maligant tumours of uncertain origin with immunohistological techniques. Lancet origin with 1985 ;i:1302-5.

5 DeLellis RA, Dayal Y. The role of immunocytochemistry in the diagnosis of poorly differentiated malignant neoplasms. Semin Oncol 1987;14:173-92.

6 Leong AS-Y, Wright J. The contribution of immunohistochemical staining in tumour diagnosis. Histopathology 1987;11:1295-1305.

7 Harris M. Differential diagnosis of spindle cell tumours by electron microscopy: personal experience and review. Histopathology 1981;5:81-105.

8 Fisher $C$. The value of electron microscopy and immunocytochemistry in the diagnosis of soft tissue sarcomas: a study of 200 cases. Histopathology 1990;16:441-54.

9 Wick MR, Swanson PE, Manival JC. Immunohistochemica analysis of soft tissue sarcomas: comparison with electron microscopy. Appl Pathol 1988;6:169-96.

10 Norton AJ, Thomas JA, Isaacson PG. Cytokeratin-specific monoclonal antibodies are reactive with tumours of smooth muscle derivation: an immunocytochemical and biochemical study using antibodies to intermediate filament cytoskeletal proteins. Histopathology 1987;11: 487-99.

11 Miettinen M. Immunoreactivity for cytokeratin and epithelial membrane antigen in leiomyosarcomas. Arch Patho Lab Med 1988;112:637-40.

12 Gray MH, Rosenberg AE, Dickersin GR, Bhan AK Cytokeratin expression in epithelioid vascular neoplasms. Hum Pathol 1990;21:212-7.

13 Stamp GW. The rational use of monoclonal antibodies in tumour diagnosis. Archiv für Geschwultstforchung 1990;60:71-82. 